\title{
Development of Shooting Method for Impact Systems*
}

\author{
Yutaka YOSHITAKE ${ }^{* *}$, Atsuo SUEOKA***, Tatsuhiko MIYUKI ${ }^{* * * *}$, Takashi HAMANO ${ }^{\dagger}$, \\ Souichirou KITAYAMA ${ }^{\dagger \dagger}$ and Sou TAMURA ${ }^{\dagger \dagger \dagger}$
}

\begin{abstract}
A shooting method is a very powerful numerical method to obtain periodic solutions of nonlinear systems. However, as a variational equation of motion is needed in the shooting method and it is very difficult to obtain it in the impact systems, the shooting method for impact systems has not been developed. In this report, a shooting method for impact systems is presented by solving this problem of variational equation. Namely, the variational equation with the delta function and its differentiation is derived. It is shown that the calculation speed of this method is very fast and complicated periodic solutions are easily obtainable in high accuracy. The stabilities of periodic solutions obtained in the shooting method are in good accordance with those obtained by the analytical method. The discontinuities in the stability of the periodic solutions are shown using characteristic multiplier. Lyapunov exponents are also calculated by applying the integral technique of variational equation.
\end{abstract}

Key Words: Nonlinear Vibration, Forced Vibration, Computer Aided Analysis, Shooting Method, Periodic Solution, Chaos, Lyapunov Exponents

\section{Introduction}

Various impact vibrations have been observed in many machines and structures: for example, the vibrations can be observed in impact damper ${ }^{(1)-(5)}$ where collision is essential, in machines utilizing impact vibrations ${ }^{(6),(7)}$ and in gear rattling of the driven systems ${ }^{(8)},(9)$. Moreover, the collision vibrations of rotors $^{(10)}$, a piping system $^{(11)}$ and fuel rods used in nuclear power generators ${ }^{(12)}$ at the time of an earthquake have been researched. The theoretical treatings of the collision in these researches are divided into two categories. One is the theory that treats a collision phenomenon as an instantaneous phenomenon using

* Received 19th January, 2004 (No. 04-4029)

** Department of Structural Engineering, Nagasaki University, 1-14 Bunkyoumachi, Nagasaki 852-8521, Japan. E-mail: yoshitak@st.nagasaki-u.ac.jp

*** Department of Intelligent Machinery and Systems, Kyushu University, 6-1-1 Hakozaki, Higashi-ku, Fukuoka 812-8581, Japan

**** Hitachi Engineering Co., Ltd., 3-2-1 Miyukimachi, Hitachi, Ibaraki 317-0073, Japan

$\dagger$ Aichi Machine Industry Co., Ltd., 4-16 Nozeki 5chome, Minato-ku, Nagoya 455-0845, Japan

† Seiryo Engineering Co., Ltd., 7-1-14 Watamiyadoori, Hyogo-ku, Khobe 652-0863, Japan

†† Niigata Engineering Co., Ltd., 10-1 Kamata-Honcho 1 chome, Ohta-ku, Tokyo 144-8639, Japan a coefficient of restitution, and the other is the one that describes the collision phenomena using linear or nonlinear restoring force. Although an actual collision happens within a limited time like the latter theory, since the time is very short, many numerical computation results or analytical results by the former theory are qualitatively in agreement with the experimental results. For example, these are the following researches: researches ${ }^{(13),(14)}$ that treat the nonlinear dynamics phenomenon caused by impact , many researches ${ }^{(2),(3),(5)}$ about impact dampers, researches ${ }^{(6),(7)}$ about machines utilizing impact vibration, and so on. Furthermore, the numerical or analytical results correspond quantitatively with the experimental results in some ${ }^{(5),(6),(13)}$ of these researches. Therefore, it can be said that the method of dealing with impact as an instantaneous phenomenon is the method that can fully explain an impact vibration.

In the history of research of impact vibration, many researches about bifurcation leading to chaos have been made in recent years. Especially, grazing bifurcation where a periodic solution suddenly bifurcates to chaos has been reported. However, like the bifurcation in an oscillating system accompanied by friction, the argument, not from the viewpoint of bifurcation from a periodic solution to chaos but from the viewpoint of the discontinuity in the stability of the solution containing the bifurcation from one periodic solution to the other periodic solution 
is required. Moreover, for example, regarding the periodic vibration generated in a two-degree-of-freedom system with an impact damper, only the simple periodic solution with several times of collisions is calculated by connecting the analytical solutions. The universal method by which a solution with any number of times of a collision can be obtained should be applied to such system.

From the above-mentioned situation, development of the method by which all kind of periodic solutions can be precisely obtained in a short time where stability of the solution can also be judged, is desired, in the analysis of the impact system whose impact is described by the instantaneous phenomena.

The best method is the shooting method. The shooting method ${ }^{(15)-(17)}$ is used as one of useful numerical methods to obtain complicated periodic solutions of nonlinear systems, or to investigate bifurcations of periodic solutions in recent years: This is the combined method using ordinary numerical integral method and the Newton method. Therefore, the highly precise solutions, even the unstable solutions, are obtainable within a short calculation time by using this method. The authors have developed a shooting method ${ }^{(18)}$ to be applicable on a system with discontinuous force such as a frictional force, for example. Moreover they submitted the new shooting method $^{(19)}$ for piecewise linear systems which uses analytical solutions on each of the linear regions instead of numerically integrated solutions. However, the application of the shooting method to the impacting system is difficult and the shooting method for the impacting system has not yet been developed, because it is unknown how instantaneous change of momentum is introduced to an equation of motion and its variational equation.

In this paper, the authors have proposed a shooting method for impact vibration systems that contain not only discontinuous force but also the discontinuous changes in momentum. This method is developed highly from shooting methods ${ }^{(18),(19)}$ that the author previously developed for systems with discontinuous forces. The equation of motion in the impact vibration system is expressed by an equation with a step function and a delta function, and its variational equation is expressed by an equation with a delta function and its differentiation; therefore, by solving the problem of calculating the process of variation at an instance of impact, application of the shooting method to the impact vibration system becomes possible. Moreover, by adapting this technique of calculating variational equations, a method to calculate the Lyapunov exponent becomes possible.

\section{Numerical Analysis Method}

\subsection{Algorithm of shooting method for impact sys- tem}

An outline of calculation procedures in the shooting method is shown below. In this method, we treat the impact phenomenon where the sign of the velocity changes instantaneously and it is expressed by using a coefficient of restitution. It is assumed that there is no nonlinearity without impact for convenience of treatment. In application, such as the above-mentioned shooting method ${ }^{(5)}$, the method consisting of the Newton-Raphson method and analytical solutions of individual linear equations of motion is formulized. It is possible to obtain a periodic solution in a much shorter calculation time by using this new shooting method (hereafter called the Exact + Shooting method) than the ordinary shooting method.

The equation of motion is expressed as follows:

$$
\dot{y}=f(y, \tau)
$$

Here, $\tau$ is time, $\cdot$ denotes $d / d \tau$, and $\boldsymbol{y}, \boldsymbol{f}$ are the following $n$-dimensional vectors.

$$
\begin{aligned}
& \boldsymbol{y}={ }^{t}\left(y_{1}, y_{2}, \cdots, y_{n}\right), \\
& \boldsymbol{f}={ }^{t}\left(f_{1}, f_{2}, \cdots, f_{n}\right)
\end{aligned}
$$

where, upper-subscript $t$ expresses a transposition sign. Equation (1) consists of equations of motion which are linear between collisions, and $f$ contains step functions and delta functions. As the impact phenomenon which is expressed by using a coefficient of restitution is considered, the terms of Eq. (1) are expressed with the following equation:

$$
f_{i}=f_{i}^{0}+\sum_{j=1}^{m_{i}} r_{i}^{j} u\left(g_{i}^{j}-c_{i}^{j}\right)+\sum_{j=1}^{m_{i}} s_{i}^{j} \delta\left(g_{i}^{j}-c_{i}^{j}\right)
$$

where, $u(\cdot)$ is step function, $\delta(\cdot)$ is Dirac's delta function and $j=1,2, \ldots, m_{i}$. In the Eq. (2), $f_{i}$ is expressed as the sum of continuous function $f_{i}^{0}$ and $m_{i}$ pieces of step functions and delta functions, and $f_{i}$ has the discontinuous quantity of $r_{i}^{j}(\boldsymbol{y}, \tau)$ and $s_{i}^{j}(\boldsymbol{y}, \tau)$ at the moment of $g_{i}^{j}(\boldsymbol{y}, \tau)=c_{i}^{j}$ which shows any condition where impact happens. The step function and the delta function, which are introduced by a collision, correspond to the viscous force and impact force respectively. Therefore, these discontinuous changes shall be produced here at the same time. However, in numerical computation, we can treat the case where those forces act individually.

On the other hand, the variational equation corresponding to the equation of motion (1) becomes the following:

$$
\dot{\eta}=A \boldsymbol{\eta}
$$

where,

$$
\begin{aligned}
\boldsymbol{\eta}={ }^{t}\left(\eta_{1}, \eta_{2}, \cdots, \eta_{n}\right), \quad \boldsymbol{A}=\frac{\partial \boldsymbol{f}}{\partial \boldsymbol{y}}=\left[A_{i k}\right] \\
A_{i k}=\partial f_{1} / \partial y_{k} \\
=\frac{\partial f_{i}^{0}}{\partial y_{k}}+\sum_{j=1}^{m_{i}}\left\{\frac{\partial r_{i}^{j}}{\partial y_{k}} u\left(g_{i}^{j}-c_{i}^{j}\right)+r_{i}^{j} \delta\left(g_{i}^{j}-c_{i}^{j}\right) \frac{\partial g_{i}^{j}}{\partial y_{k}}\right. \\
\left.+\frac{\partial s_{i}^{j}}{\partial y_{k}} \delta\left(g_{i}^{j}-c_{i}^{j}\right)+s_{i}^{j} \frac{\partial \delta\left(g_{i}^{j}-c_{i}^{j}\right)}{\partial\left(g_{i}^{j}-c_{i}^{j}\right)} \frac{\partial g_{i}^{j}}{\partial y_{k}}\right\}
\end{aligned}
$$


Initial value $\boldsymbol{y}^{0}={ }^{t}\left(y_{1}(0), y_{2}(0), \cdots, y_{n}(0)\right)$ at $\tau=$ 0 is assumed, connecting the analytical solution of each linear region repeatedly, then the solution $\boldsymbol{y}^{1}=$ ${ }^{t}\left(y_{1}(T), y_{2}(T), \cdots, y_{n}(T)\right)$ at $\tau=T$ after one period is calculated. The conditions of a periodic solution will serve as the following equation:

$$
y^{1}-y^{0}=0
$$

Considering that $\boldsymbol{y}^{1}$ is calculated by connecting the solution of each linear region from assumed $\boldsymbol{y}^{0}$, it can be said that $\boldsymbol{y}^{1}$ is the function of $\boldsymbol{y}^{0}$. Therefore, obtaining a periodic solution is equivalent to finding $\boldsymbol{y}^{0}$ which satisfies Eq. (5). If the Newton-Raphson method is applied to Eq. (5), the equation for repetition calculation becomes the following:

$$
\left(\boldsymbol{B}-\boldsymbol{I}_{n}\right) \tilde{\boldsymbol{y}}=\boldsymbol{y}^{0}-\boldsymbol{y}^{1}
$$

Where, $\tilde{\boldsymbol{y}}$ is the amount of correction for the next repetition (the corrected value is $\boldsymbol{y}^{0}+\tilde{\boldsymbol{y}}$ ), and $\boldsymbol{B}$ is constructed by the solutions of variational equations $\boldsymbol{\eta}_{i}(T)=$ ${ }^{t}\left(\eta_{1}(T), \eta_{2}(T), \cdots, \eta_{n}(T)\right)(i=1, \cdots, n)$, which are calculated from the initial values $\boldsymbol{\eta}_{i}(0)={ }^{t}(0,0, \cdots, 0,1,0, \cdots, 0,0)(i$-th element is unit, $i=1, \cdots, n$ ), i.e., unit vectors which form a unit matrix. This is called the basic solution matrix:

$$
\boldsymbol{B}=\left(\boldsymbol{\eta}_{1}(T), \boldsymbol{\eta}_{2}(T), \cdots, \boldsymbol{\eta}_{n}(T)\right)
$$

For example, the convergence of a solution is judged as follows by using relative error $\varepsilon_{1}$ between initial values and the calculated values after one period $(i=1,2, \ldots, n)$ :

$$
\begin{cases}\left|\left\{y_{i}(T)-y_{i}(0)\right\} / y_{i}(0)\right| \leq \varepsilon & : y_{i}(0) \neq 0 \\ \left|y_{i}(T)-y_{i}(0)\right| \leq \varepsilon & : y_{i}(0)=0\end{cases}
$$

The flow chart of this Exact + Shooting method is shown in Fig. 1.

When the periodic solution is obtained, the stability of a periodic solution is judged as follows: If all the absolute values of the eigenvalues (these are called characteristic multipliers) of matrix $\boldsymbol{B}$ are smaller than unit, the solution is judged stable. If at least one of the absolute values is larger than unit, the solution is judged unstable.

\subsection{Processing of variations at colliding time}

If the time $\tau$ when a discontinuous function $f_{i}$ in Eq. (1) reaches the colliding point is set to $\tau_{0}$, the following equation is realized:

$$
g_{i}^{j}\left\{\boldsymbol{y}\left(\tau_{0}\right), \tau_{0}\right\}=c_{i}^{j}
$$

Defining $\varepsilon_{0}$ as minute positive value, next minute section is considered.

$$
\tau_{0}^{-}=\tau_{0}-\varepsilon_{0} \leq \tau \leq \tau_{0}
$$

Taking into consideration only a delta function and its differentiation in Eq. (4), the variational equation of this section can be approximated by the following equation:

$$
d \boldsymbol{\eta} / d \tau=\left(0, \cdots, \frac{d \eta_{i}}{d \tau}, \cdots, 0\right)
$$

where

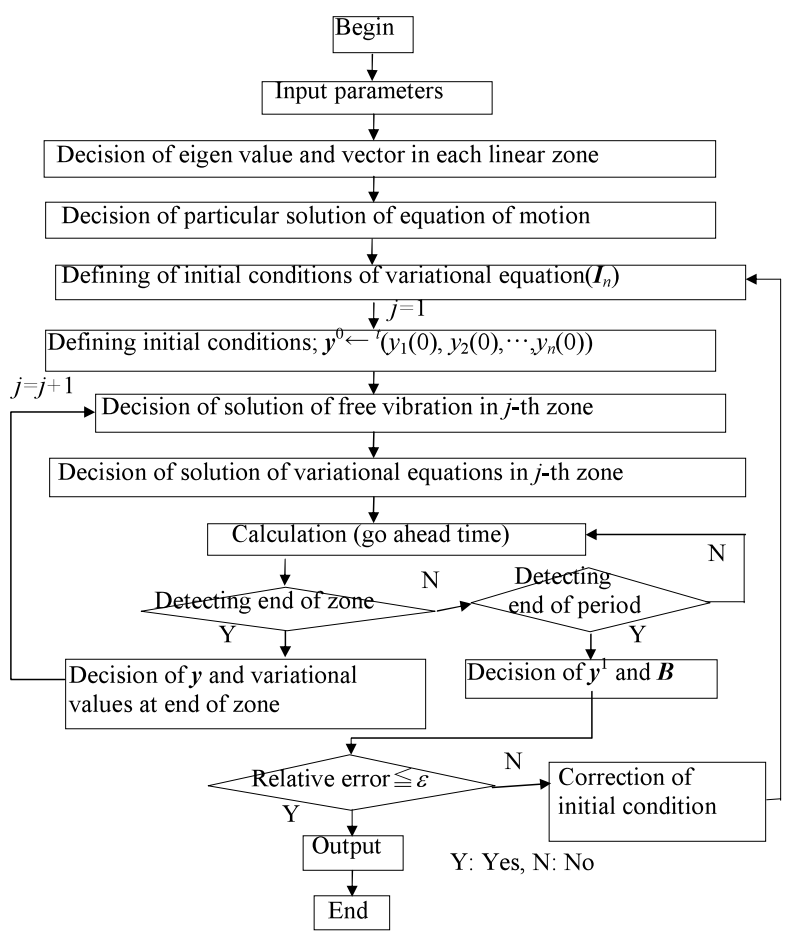

Fig. 1 Flow chart of shooting method using analytical solutions (Exact+Shooting method)

$$
\begin{aligned}
\frac{d \eta_{i}}{d \tau}= & \left\{r_{i}^{j} \delta\left(g_{i}^{j}-c_{i}^{j}\right)+s_{i}^{j} \frac{\partial \delta\left(g_{i}^{j}-c_{i}^{j}\right)}{\partial\left(g_{i}^{j}-c_{i}^{j}\right)}\right\} \sum_{k=1}^{n} \frac{\partial g_{i}^{j}}{\partial y_{k}} \eta_{k} \\
& +\delta\left(g_{i}^{j}-c_{i}^{j}\right) \sum_{k=1}^{n} \frac{\partial s_{i}^{j}}{\partial y_{k}} \eta_{k}
\end{aligned}
$$

A collision is taken into consideration in the variational equation by integrating Eq. (11) through the minute section of Eq. (9). The integration of the variational equation in this minute section can be calculated by applying the following formulas, namely, the integral of delta function and its differentiation, to the Eq. (11):

$$
\begin{aligned}
& \int_{-\infty}^{\infty} \delta(\tau) d \tau=1 \\
& \int_{-\infty}^{\infty} \delta^{\prime}(\tau) f(\tau) d \tau=-\int_{-\infty}^{\infty} \delta(\tau) f^{\prime}(\tau) d \tau=-f^{\prime}(0)
\end{aligned}
$$

Moreover, a judgment of discontinuous point attainment is performed by deciding the permissible error as follows:

$$
\left|g_{i}^{j}\left\{\boldsymbol{y}\left(\tau_{0}^{-}\right), \tau_{0}^{-}\right\}-c_{i}^{j}\right|<\varepsilon_{1}
$$

\subsection{Calculation of a Lyapunov exponent}

The Lyapunov exponent is used as an index that judges whether a phenomenon is chaos or not. For a calculation technique of the Lyapunov exponent, there is the method of Shimada-Nagashima ${ }^{(20)}$, which performs normalization of Gram-Schmidt for every suitable time of numerical integration of the variational equation. The authors improved this method to be applicable to a system with discontinuous characteristics ${ }^{(18)}$. On the other hand, since the processing of variation is difficult in the impact system, there is no example that calculates all 
Lyapunov exponents of impact system. However, if the above-mentioned technique of integrating the variational equation in the shooting method is used as it is, all Lyapunov exponents are calculable similar to the method of Shimada-Nagashima ${ }^{(20)}$.

\section{Application to Single-Degree-of-Freedom Impact System}

\subsection{Processing of variational equation}

In order to actually apply the shooting method to an impact system, it is important that we take into consideration the instantaneous change in the velocity that arises with collision. As an example, the case of the singledegree-of-freedom system is concretely explained below.

Since it is easy, the single-degree-of-freedom system expressed with the following non-dimensional equation of motion is considered:

$$
y^{\prime \prime}+2 \gamma y^{\prime}+y=F(\tau)
$$

where, $y$ is a non-dimensional displacement, $\gamma$ is a damping ratio, $\Delta$ is the non-dimensional clearance between the particle and the wall (as example, in the Fig. 3 shown later, $\Delta$ means the ratio $d / z_{0}$ of the clearance $d$ and the amplitude of the base excitation $z_{0}$ ), $\tau$ is non-dimensional time, $F(\tau)$ is the external force, and ${ }^{\prime}=d / d \tau$.

Now, when $y$ becomes $\Delta$ from the state of $y<\Delta$ at the time $\tau=\tau_{0}$ and the particle collides with a wall, the condition of a collision and the relation of the velocities before and after collision are shown as the following equations:

$$
\begin{aligned}
& g_{i}^{j}(\boldsymbol{y}, \tau)-c_{i}^{j}=y\left(\tau_{0}\right)-\Delta=0 \\
& y^{\prime}\left(\tau_{0}^{+}\right)=-e y^{\prime}\left(\tau_{0}^{-}\right)
\end{aligned}
$$

where, $e$ is a coefficient of restitution and upper subscripts - and + show just before and just after a collision, respectively. Introducing a step function to Eq. (17), the velocity between $\tau_{0}^{-}<\tau<\tau_{0}^{+}$is expressed by the following equation:

$$
y^{\prime}(\tau)=y^{\prime}\left(\tau_{0}^{-}\right)-(1+e) y^{\prime}\left(\tau_{0}^{-}\right) u(y-\Delta)
$$

Here, in fact, although $y$ is not larger than $\Delta$, the step function is used for convenience.

Next, in the moments just before and after a collision, since the displacement and external force do not change, the acceleration just after a collision becomes as follows from Eq. (15):

$$
y^{\prime \prime}\left(\tau_{0}^{+}\right)=y^{\prime \prime}\left(\tau_{0}^{-}\right)-2 \gamma\left\{y^{\prime}\left(\tau_{0}^{+}\right)-y^{\prime}\left(\tau_{0}^{-}\right)\right\}
$$

The following equation is obtained from Eqs.(18) and (19).

$$
y^{\prime \prime}(\tau)=y^{\prime \prime}\left(\tau_{0}^{-}\right)+2 \gamma(1+e) y^{\prime}\left(\tau_{0}^{-}\right) u(y-\Delta)
$$

On the other hand, if the impact force by the collision is placed with $F_{c}$, since the change of momentum by the collision is equal to impulse by impact force, the following equation is obtained from Eq. (18):

$$
\int_{\tau_{0}^{-}}^{\tau_{0}^{+}} F_{c} d \tau=y^{\prime}\left(\tau_{0}^{+}\right)-y^{\prime}\left(\tau_{0}^{-}\right)=-(1+e) y^{\prime}\left(\tau_{0}^{-}\right) u(y-\Delta)
$$

Therefore, impact force $F_{c}$ becomes as follows:

$$
\begin{aligned}
F_{c}(\tau) & =d\left\{-(1+e) y^{\prime}\left(\tau_{0}^{-}\right) u(y-\Delta)\right\} / d \tau \\
& =-(1+e) y^{\prime}\left(\tau_{0}^{-}\right)^{2} \delta(y-\Delta)
\end{aligned}
$$

By adding impact force $F_{c}$ to Eq. (20), the acceleration between $\tau_{0}^{-}<\tau<\tau_{0}^{+}$is shown as follows:

$$
\begin{aligned}
y^{\prime \prime}(\tau)= & y^{\prime \prime}\left(\tau_{0}^{-}\right)+2 \gamma(1+e) y^{\prime}\left(\tau_{0}^{-}\right) u(y-\Delta) \\
& -(1+e) y^{\prime}\left(\tau_{0}^{-}\right)^{2} \delta(y-\Delta)
\end{aligned}
$$

As mentioned above, the velocity and the acceleration in $\tau_{0}^{-}<\tau<\tau_{0}^{+}$can be expressed by the following equations:

$$
\begin{aligned}
y^{\prime}(\tau)= & y^{\prime}\left(\tau_{0}^{-}\right)-(1+e) y^{\prime}\left(\tau_{0}^{-}\right) u(y-\Delta) \\
y^{\prime \prime}(\tau)= & y^{\prime \prime}\left(\tau_{0}^{-}\right)+2 \gamma(1+e) y^{\prime}\left(\tau_{0}^{-}\right) u(y-\Delta) \\
- & (1+e) y^{\prime}\left(\tau_{0}^{-}\right)^{2} \delta(y-\Delta)
\end{aligned}
$$

Next, when it sets with $y_{1}=y, y_{2}=y^{\prime}$, the equation of motion which contains the collision arising in $\tau_{0}^{-}<\tau<\tau_{0}^{+}$ can be expressed as follows from the above equations:

$$
\frac{d y}{d \tau}=f
$$

where,

$$
\begin{aligned}
\boldsymbol{y}= & \left(y_{1}, y_{2}\right), \quad \boldsymbol{f}={ }^{t}\left(f_{1}, f_{2}\right) \\
f_{1}= & -(1+e) y_{2}\left(\tau_{0}^{-}\right) u\left\{y_{1}(\tau)-\Delta\right\} \\
f_{2}= & 2 \gamma(1+e) y_{2}\left(\tau_{0}^{-}\right) u\left\{y_{1}(\tau)-\Delta\right\} \\
& -(1+e) y_{2}\left(\tau_{0}^{-}\right)^{2} \delta\left\{y_{1}(\tau)-\Delta\right\}
\end{aligned}
$$

The variational equation is obtained from Eqs. (25) and (26). Moreover, the relationship between the variation before collision and that after collision is expressed with the following equation by carrying out the integrations of $\delta$ function and that of differentiation of delta function.

$$
\begin{aligned}
\eta_{1}\left(\tau_{0}^{+}\right)=\eta_{1}\left(\tau_{0}^{-}\right)+\Delta \eta_{1} \\
\eta_{2}\left(\tau_{0}^{+}\right)=\eta_{2}\left(\tau_{0}^{-}\right)+\Delta \eta_{2} \\
\Delta \eta_{1}=-(1+e) \eta_{1}\left(\tau_{0}^{-}\right) \\
\Delta \eta_{2}=-(1+e)\left\{y_{1}\left(\tau_{0}^{-}\right)-F\left(\tau_{0}^{-}\right)\right\} \eta_{1}\left(\tau_{0}^{-}\right) / y_{2}\left(\tau_{0}^{-}\right) \\
\quad-(1+e) \eta_{2}\left(\tau_{0}^{-}\right)
\end{aligned}
$$

The coefficient of restitution is treated as constant value independent of colliding velocity here. On the other hand, we confirmed that the shooting method could be applied to the case that the coefficient of restitution was expressed as a function of the colliding velocity.

\subsection{Impact vibration of a ball on vibrating table}

The impact vibration of a ball on a vibrating table is considered as shown in Fig. 2. Some periodic solutions are shown using analytical solution method by Luo and $\operatorname{Han}^{(21)}$. The above mentioned shooting method is applied to this system, and the validity of this technique is proved by comparing the results obtained with those by an analytical solution method ${ }^{(21)}$. The comparison of the stability 


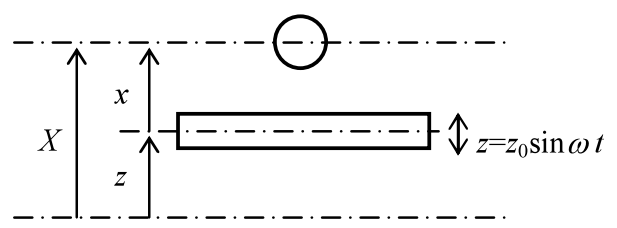

Fig. 2 Model of a bouncing ball

of steady-state solutions, to which processing of a variational equation affects directly, is especially important.

The absolute displacements of a ball and a vibrating table are defined as $X$ and $z=z_{0} \sin \omega t$ respectively. If relative displacement is set to $x=X-z$, the equation of motion of the system becomes as follows:

$$
\ddot{x}=z_{0} \omega^{2} \sin \omega t-g
$$

When the above equation is formed non-dimensionally, it is shown as follows:

$$
y^{\prime \prime}=K \sin \tau-1
$$

where,

$$
\tau=\omega t, \quad{ }^{\prime}=d / d \tau, \quad y=x /\left(g / \omega^{2}\right), \quad K=z_{0} /\left(g / \omega^{2}\right)
$$

The amount of steps of variations equivalent to Eqs. (29) and (30) becomes as follows:

$$
\begin{aligned}
\Delta \eta_{1}= & -(1+e) \eta_{1}\left(\tau_{0}^{-}\right) \\
\Delta \eta_{2}= & -(1+e)\left(1-K \sin \tau_{0}^{-}\right) \eta_{1}\left(\tau_{0}^{-}\right) / y_{2}\left(\tau_{0}^{-}\right) \\
& -(1+e) \eta_{2}\left(\tau_{0}^{-}\right)
\end{aligned}
$$

where, $y_{1}=y, y_{2}=y^{\prime}$.

Luo and Han ${ }^{(21)}$ calculated analytically the simple solution which performs one collision in $n$ cycles and judged its stability. The stable condition of the periodic solution is expressed with the following equation:

$$
n \pi\left(\frac{1-e}{1+e}\right)<K<\sqrt{n^{2} \pi^{2}\left(\frac{1-e}{1+e}\right)^{2}+4 \frac{\left(1+e^{2}\right)^{2}}{(1+e)^{4}}}
$$

Setting the coefficient of restitution $e=0.9$ and considering the stable solution which performs one collision in 1 cycle, the range of amplitude $K$ of non-dimensional acceleration of table is expressed as follows from Eq. (36):

$$
0.16534698176<K<1.01631071230
$$

The result calculated by this technique is shown in Table 1, where, the discontinuous point attainment permissible error $\varepsilon_{2}$ is $1 \times 10^{-13}$. Hereafter, in this paper, the value of this $\varepsilon_{2}$ is used in all examples. The convergence accuracy of a solution shown in Eq. (7) is $\varepsilon_{1}=1 \times 10^{-10} \cdot \lambda_{1}$ and $\lambda_{2}$ in the table are characteristic multipliers and serves as the real number here. It turns out that the values of $K$, when the absolute value of a characteristic multiplier exceeds 1 and the stability of a solution changes, agree up to 10 figures with the boundary values of stability by Luo and $\operatorname{Han}^{(21)}$. This means that processing of the variational
Table 1 Boundary of stability $(e=0.9)$

(a) Upper boundary

\begin{tabular}{|l|l|l|}
\hline \multicolumn{1}{|c|}{$K$} & \multicolumn{1}{c|}{$\lambda_{1}$} & \multicolumn{1}{c|}{$\lambda_{2}$} \\
\hline 1.016 & -0.8150084435 & -0.9938547342 \\
\hline 1.0163 & -0.8101672703 & -0.9997935361 \\
\hline 1.016310 & -0.8100111112 & -0.9999862827 \\
\hline 1.01631071 & -0.8100000359 & -0.9999999556 \\
\hline 1.0163107123 & -0.8100000000 & -0.9999999999 \\
\hline 1.0163107124 & -0.8099999985 & -1.0000000018 \\
\hline 1.01631072 & -0.8099998800 & -1.0000001482 \\
\hline 1.016311 & -0.8099955127 & -1.0000055399 \\
\hline 1.0164 & -0.8086197094 & -1.0017069713 \\
\hline 1.017 & -0.7999175716 & -1.0126043342 \\
\hline 1.01631071230 & $\leftarrow$ Boundary of stability (By Luo and \\
& Han $^{(21)}$ ) \\
\hline
\end{tabular}

(b) Lower boundary

\begin{tabular}{|l|l|l|}
\hline \multicolumn{1}{|c|}{$K$} & \multicolumn{1}{c|}{$\lambda_{1}$} & \multicolumn{1}{c|}{$\lambda_{2}$} \\
\hline 0.1653469818 & 0.9999380597 & 0.8100501747 \\
\hline 0.16534699 & 0.9990044310 & 0.8108072145 \\
\hline 0.165347 & 0.9985152716 & 0.8112044182 \\
\hline 0.1654 & 0.8974414625 & 0.8974414625 \\
\hline 0.166 & 0.8784489703 & 0.8784489703 \\
\hline 0.16534698176 & $\begin{array}{l}\leftarrow \text { Boundary of stability (By Luo and } \\
\operatorname{Han}^{(21)} \text { ) }\end{array}$ \\
\hline
\end{tabular}

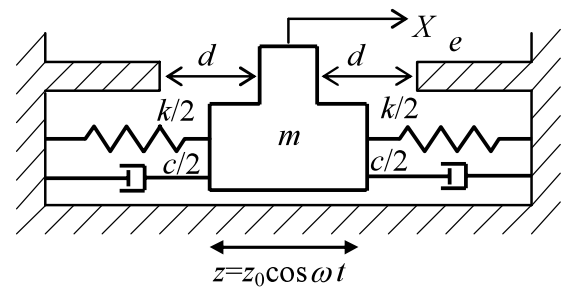

Fig. 3 Model of forced system with two sided impacts

equation at the time of a collision of this technique is correct. Moreover, if the amplitude $K$ of non-dimensional acceleration of table is made less than the value in Table 1 (b), the periodic solution cannot be realized, because the amplitude $K$ is less than the value required to carry out vibration which performs one collision in 1 cycle.

\subsection{Impact vibration of base excitation system}

Imamura et al. ${ }^{(22)}$ researched the impact vibration of the single-degree-of-freedom base excitation system, and $1 / n$-th sub-harmonic vibration and bifurcation to chaos are treated. Moreover, the existence of grazing bifurcation ${ }^{(23)}$ where contacts arise at a relative velocity of zero is also reported in the impact system.

In this study, the same system as Imamura et al. ${ }^{(22)}$ is taken as an example, and discontinuities of characteristic multipliers in grazing bifurcation are shown using shooting method. The model of the impact system, which has the rigid collision walls of coefficient of restitution $e$ on both sides, is shown in Fig. 3. Specifically, the mass of particle is $m$, the absolute displacement is $X$, and the width of clearance is $d$. There exist the springs with spring con- 
stant $k / 2$ on both sides of the particle, the dampings with viscous coefficient $c / 2$ on both sides, and the base excitation of $z=z_{0} \cos \omega t$ acts.

The equation of motion of the particle is expressed as follows:

$$
m \ddot{x}+c \dot{x}+k x=m \omega^{2} z_{0} \cos \omega t
$$

where $x=X-z$ is the relative displacement of the particle. The non-dimensional equation of motion becomes as follows.

$$
y^{\prime \prime}+2 \gamma y^{\prime}+y=v^{2} \cos v \tau
$$

where,

$$
\begin{aligned}
& \omega_{n}=\sqrt{k / m}, \quad v=\omega / \omega_{n}, \quad \tau=\omega_{n} t, \quad '=d / d \tau \\
& \gamma=c / 2 \sqrt{m k}, \quad y=x / z_{0}, \quad \Delta=d / z_{0}
\end{aligned}
$$

The amount of steps of variations which are equivalent to Eqs. (29) and (30), serves as the following equation:

$$
\begin{aligned}
\Delta \eta_{1}= & -(1+e) \eta_{1}\left(\tau_{0}^{-}\right) \\
\Delta \eta_{2}= & -(1+e)\left\{y_{1}\left(\tau_{0}^{-}\right)-v^{2} \cos \left(v \tau_{0}^{-}\right)\right\} \eta_{1}\left(\tau_{0}^{-}\right) / y_{2}\left(\tau_{0}^{-}\right) \\
& -(1+e) \eta_{2}\left(\tau_{0}^{-}\right)
\end{aligned}
$$

where, $y_{1}=y, y_{2}=y^{\prime}$.

The bifurcation diagram, the collision velocity characteristics, and the characteristic multiplier are shown in Fig. 4 , where non-dimensional clearance $\Delta=1$, coefficient of restitution $e=0.7$ and damping ratio $\gamma=0.01$ are used. The ordinate of Fig. 4(a) is the displacement when the phase of base excitation is zero, and that of Fig. 4 (b) is the absolute value of the velocity just before collision. When multiple collisions occur, the greatest velocity is adopted. The sign showing the kind of solution in each figure is defined as follows: $1 / n$ expresses the order of sub-harmonic vibration, $\mathrm{O}$ means that the Fourier series of the solution contains only the odd order, E means that the series contains both the odd order and the even order, and the rightmost number expresses the number of collisions per period of the solution. For example, 1/1-O2 means that solution is the harmonic vibration composed of only the odd order of the Fourier series and contains two collisions per period. Moreover, it is related to the characteristic multiplier shown in Fig. 4 (c), the larger one concerning the absolute value which decides the stability of periodic solution is shown. $\lambda_{r}$ in Fig. 4 (c) means that the characteristic multiplier is complex and its real part is shown in the figure. The bifurcation diagram of Fig. 4 (a) plots the periodic solution and chaos which were converged only by repeating the connection of analytical solutions, without using the shooting method. On the other hand, the impact velocity of Fig. 4 (b) is calculated using the shooting method. Although one ordinate is displacement and the other is velocity, all regions of solution agree completely in both figures.

Adopting the first figure of once converged solution 1/1-O2 of $v=1.2$ in Fig. 4 (b) as the initial value, the periodic solution of $1 / 1-\mathrm{O} 2$ is calculated from $v=1.2$ to

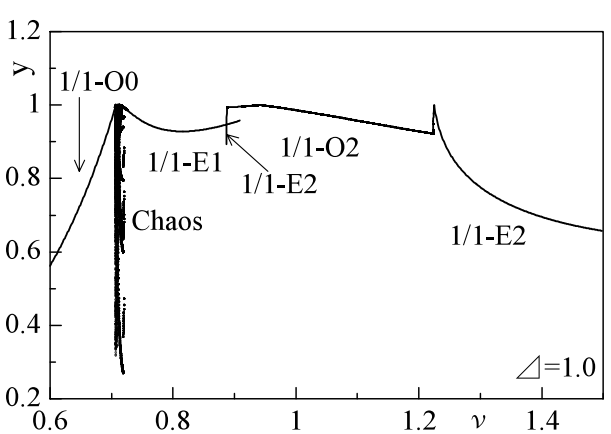

(a) Bifurcation diagram

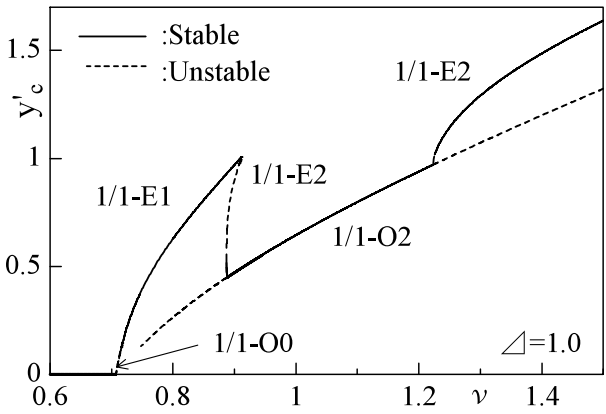

(b) Contact velocity

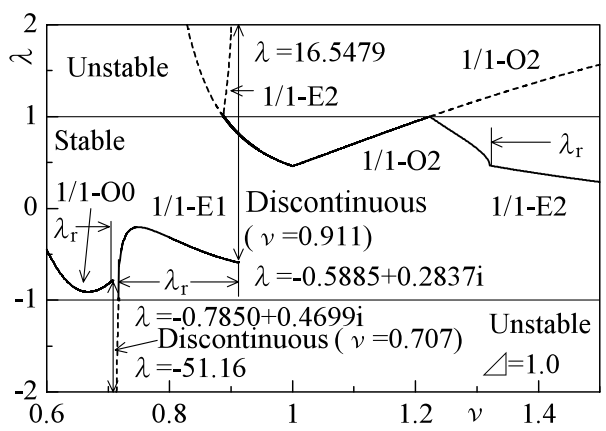

(c) Characteristic multiplier

Fig. 4 Characteristic of forced system $(e=0.7, \Delta=1, \gamma=0.01)$

Table 2 Comparison of calculation time $(e=0.7, \Delta=1, \gamma=$ $0.01, v=1.2 \rightarrow 1.0)$

\begin{tabular}{|c|c|c|c|}
\hline$v$ & Method of calculation & $\begin{array}{c}\text { Initial number } \\
\text { of division }\end{array}$ & $\begin{array}{c}\text { Calculation } \\
\text { time (s) }\end{array}$ \\
\hline \multirow{3}{*}{$\begin{array}{c}1.2 \\
\downarrow\end{array}$} & RKG - Not shooting & \multirow{2}{*}{1024} & 8.13 \\
\cline { 2 - 2 } 1.0 & RKG+Shooting & & 0.66 \\
\cline { 2 - 2 } & Exact - Not shooting & \multirow{2}{*}{64} & 3.23 \\
\cline { 2 - 2 } & Exact+Shooting & & 0.16 \\
\hline
\end{tabular}

$v=1.0$ by decreasing the value of $v$ in the unit width of 0.01 . A comparison of calculation times is shown in Table 2, where the convergence accuracy of a solution was set to $\varepsilon=1 \times 10^{-10}$. For this calculation, a Sun Enterprise 450 workstation, which is a UNIX machine was used, because to measure the exact calculation time is difficult in Windows machine. The minimal measuring unit of the calculation time was 0.01 second in the compiler used, the FUJITSU Fortran90 compiler 2.0. This is not small; therefore, the low calculation speed of this machine man- 
ufactured in 1998 was used intentionally to measure the calculation time precisely.

In Table 2, the calculation times taken to obtain the periodic solutions are compared. In the table, not the calculation time itself but the comparison of the calculation times is meaningful. The first method in the table, hereafter called the RKG-Not Shooting method, is the one that continues integration from the initial value using the Runge-Kutta-Gill method, without using the principle of the Newton-Raphson method. The second method, the Exact-Not Shooting method, repeats the connection of analytical solutions. The third method, the RKG+Shooting method, is the shooting method which calculates a solution in every linear term by numerical integration using the Runge-Kutta-Gill method. The last is the Exact+Shooting method mentioned in chapter 2 . The initial number of divisions in the table expresses the number of divisions of period in the first stage. That is, the value which divides the period of external force $2 \pi / v$ by this initial number of divisions is the step size in the first stages of the RungeKutta-Gill method. In the analytical solution, it is equivalent to time step size to advance time $\tau$. Although the first step size is changed in order to detect the boundary of each linear section with predetermined accuracy during the execution of each solution method, if the boundary is detected at once, the step size will be reset to the first one in the next linear section. Moreover, in the methods of Exact-Not Shooting and Exact+Shooting, the procedure which calculates a general solution (the 3rd and 4th steps in Fig. 1) is also included in the calculation time.

The usual Runge-Kutta-Gill method (RKG-Not Shooting method) takes the most time in Table 2. This method is followed by the analytical solution method (Exact-Not Shooting method) which does not correct the initial value after one period, the RKG+Shooting method and the Exact+Shooting method, in that order. The Exact + Shooting method can calculate a solution in the shortest time. The RKG+Shooting method can calculate in only one-twelfth of the time in the RKG-Not Shooting method, which does not correct initial value. Therefore, the shooting method itself is an outstanding method. The Exact+Shooting method can calculate the periodic solution in one-fourth of calculation time in the RKG+Shooting method, and it can be said that this is a very high-speed method.

There is a bifurcation from the stable solution of $1 / 1$ E1 to the unstable solution of $1 / 1-\mathrm{E} 2$ near $v=0.9$ in Fig. 4. This bifurcation is caused by the increase of collisions per cycle. At the bifurcation point, the resonance curve of impact velocity in Fig. 4(b) is turned over in the form where it sharpened, and the characteristic multiplier in Fig. 4 (c) changes discontinuously a great deal on both sides of value 1. Although such a phenomenon caused by generation of a collision was called grazing bifurca- tion $^{(23)}$, it was shown for the first time that a characteristic multiplier changes discontinuously. Grazing bifurcation means discontinuity in stability of solution. The reason a characteristic multiplier changes discontinuously is that the amount of step values of variations shown by Eqs. (41) and (42) are added by generation of a collision. Such a characteristic phenomenon is the same as that of the forced self-excited vibration system accompanied by dry friction ${ }^{(24)}$, and that of the preloaded compliance system $^{(25)}$, which the authors have worked with before. On the other hand, in a system with continuous nonlinearity like Duffing's equation, the inclinations of stable and unstable branches in resonance curve become infinite and are in agreement each other, and a characteristic multiplier changes continuously through $\lambda=1$. Also in this system, the characteristic multiplier of 1/1-O2 shown in Fig. 4 (c) is 1 and changes continuously at the stable and unstable boundaries. This is because the number of times of a collision does not change.

Next, the calculation of a Lyapunov exponent was carried out utilizing the method of ShimadaNagashima $^{(20)}$, which performs normalization of GramSchmidt after every suitable time integration of the equation of motion, and the variational equation was used. In this study, numerical integration was not performed but analytical solutions of each section are connected. Moreover, whenever a collision is generated, the step-changes of variation shown by Eqs. (41) and (42) are taken into consideration.

In this study, a direct bifurcation from $1 / 1-\mathrm{O} 0$ to chaos by grazing bifurcation ${ }^{(23)}$ is also obtained as shown in Fig. 4 (a). Here, chaos is judged using the Lyapunov exponents. The Poincare section and Lyapunov exponents of 1/1-O0 are shown in Fig. 5. Those in the case of chaos are shown in Fig. 6. In calculation of the Poincare section, chaos is calculated by connecting the analytical solutions, and the periodic solution is calculated by the shooting method and checked also by connecting an analytical solutions. On the other hand, the calculation of the Lyapunov exponents is carried out using the information of variational equation. As the results of (a) and (b) correspond well in each figure, it can be said that the processing

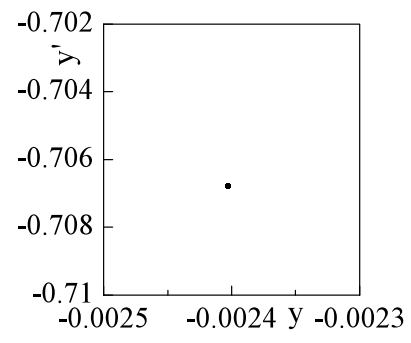

(a) Poincare section

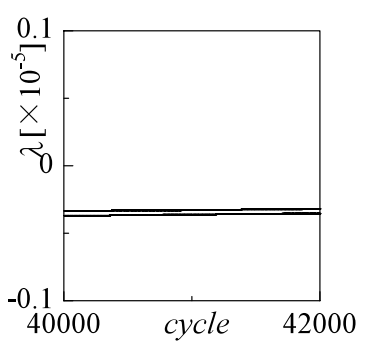

(b) Lyapunov exponents
Fig. 5 Periodic solution $1 / 1-\mathrm{O} 0(\Delta=1, v=0.7071)$ 


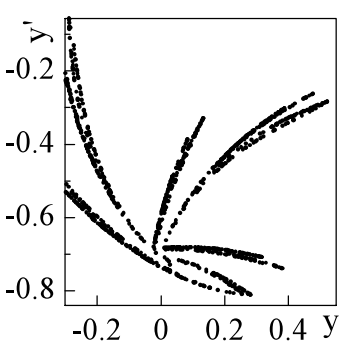

(a) Poincare section

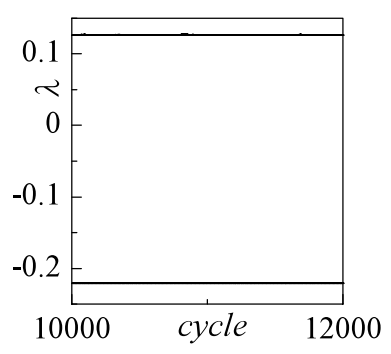

(b) Lyapunov exponents
Fig. 6 Chaos $(\Delta=1, v=0.7072)$

variational equations at a colliding point is correct, and the Lyapunov exponent is calculated correctly.

\section{Apprication to Two-Degree-of-Freedom System}

As a two-degree-of-freedom system with collision, the model of the classical impact damper system shown in Fig. 7 is considered. Specifically, the single-degree-offreedom forced system composed of mass $M$, spring constant $k$, and viscous damping coefficient $c$ is controlled by the impact damper constituted from a ball of mass $m$ and rigid walls of coefficient of restitution $e$. Masri and Caughey ${ }^{(1)}$ calculated analytically a simple solution with two collisions in one period and judged its stability. The shooting method is applied to this system. The validity of this method is proved by comparing the result of this technique with the result of the analytical solution method of Masri and Caughey ${ }^{(1)}$, especially the stability of the steady state solution in which processing of the variational equation is directly involved is compared.

The absolute displacements of main system and a ball are $x_{1}$ and $x_{2}$ respectively, the relative displacement is $x_{r}=x_{2}-x_{1}$, and the clearance between walls is $2 d$. When the external force $F \cos \omega t$ acts on the main system, the equation of motion becomes as follows:

$$
\begin{aligned}
& M \ddot{x}_{1}+c \dot{x}_{1}+k x_{1}=F \cos \omega t \\
& m \ddot{x}_{2}=0
\end{aligned}
$$

The non-dimensional equation of motion of this system is as follows:

$$
\begin{aligned}
& Y_{1}^{\prime \prime}+2 \gamma Y_{1}^{\prime}+Y_{1}=\cos v \tau \\
& Y_{2}^{\prime \prime}=0
\end{aligned}
$$

where,

$$
\begin{aligned}
& \omega_{n}=\sqrt{k / M}, v=\omega / \omega_{n}, '=d / d \tau, \tau=\omega_{n} t \\
& \gamma=c / 2 \sqrt{M k}, Y_{1}=x_{1} / \delta_{s t}, Y_{2}=x_{2} / \delta_{s t}, y_{r}=Y_{2}-Y_{1} \\
& \delta_{s t}=F / k, \Delta=d / \delta_{s t}
\end{aligned}
$$

Supposing $y_{r}= \pm \Delta$ at time $\tau=\tau_{0}$ and a ball collides with the wall of a main system, the conditions of the impact velocity between a main system and a ball is expressed by the following equations:

$$
\begin{aligned}
& Y_{1}^{\prime}\left(\tau_{0}^{+}\right)=\alpha_{1} Y_{1}^{\prime}\left(\tau_{0}^{-}\right)+\alpha_{2} Y_{2}^{\prime}\left(\tau_{0}^{-}\right) \\
& Y_{2}^{\prime}\left(\tau_{0}^{+}\right)=\alpha_{3} Y_{1}^{\prime}\left(\tau_{0}^{-}\right)+\alpha_{4} Y_{2}^{\prime}\left(\tau_{0}^{-}\right)
\end{aligned}
$$

where,

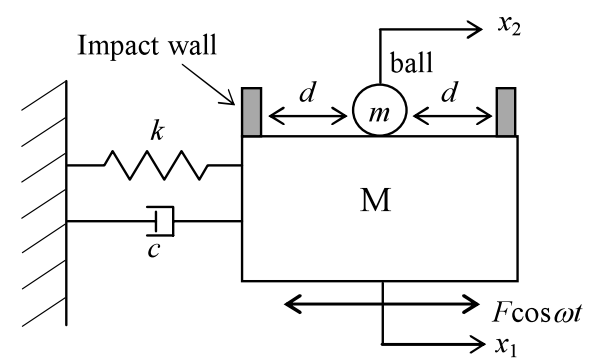

Fig. 7 Model of impact system

$$
\begin{aligned}
& \mu=m / M, \mu_{1}=1+\mu, \alpha_{1}=(1-\mu e) / \mu_{1} \\
& \alpha_{2}=\mu(1+e) / \mu_{1}, \alpha_{3}=(1+e) / \mu_{1}, \alpha_{4}=(\mu-e) / \mu_{1}
\end{aligned}
$$

The equations of motion corresponding to Eqs. (25) and (26) become as follows:

$$
\begin{aligned}
& \frac{d \boldsymbol{y}}{d \tau}=\boldsymbol{f}: \boldsymbol{y}={ }^{t}\left(y_{1}, y_{2}, y_{3}, y_{4}\right), \boldsymbol{f}={ }^{t}\left(f_{1}, f_{2}, f_{3}, f_{4}\right) \\
& f_{1}=\alpha_{2} y_{r}^{\prime}\left(\tau_{0}^{-}\right) u\left( \pm y_{r}-\Delta\right) \\
& f_{2}=-2 \gamma \alpha_{2} y_{r}^{\prime}\left(\tau_{0}^{-}\right) u\left( \pm y_{r}-\Delta\right) \pm \alpha_{2} y_{r}^{\prime}\left(\tau_{0}^{-}\right)^{2} \delta\left( \pm y_{r}-\Delta\right) \\
& f_{3}=-\alpha_{3} y_{r}^{\prime}\left(\tau_{0}^{-}\right) u\left( \pm y_{r}-\Delta\right) \\
& f_{4}=\mp \alpha_{3} y^{\prime}\left(\tau_{0}^{-}\right)^{2} \delta\left( \pm y_{r}-\Delta\right)
\end{aligned}
$$

where, complex sign is in the same order and $y_{1}=Y_{1}, y_{2}=$ $Y_{1}^{\prime}, y_{3}=Y_{2}, y_{4}=Y_{2}^{\prime}$. Moreover, the amount of steps of variations becomes as follows:

$$
\begin{aligned}
& \eta_{1}\left(\tau_{0}^{+}\right)=\eta_{1}\left(\tau_{0}^{-}\right)+\Delta \eta_{1}, \quad \eta_{2}\left(\tau_{0}^{+}\right)=\eta_{2}\left(\tau_{0}^{-}\right)+\Delta \eta_{2} \\
& \eta_{3}\left(\tau_{0}^{+}\right)=\eta_{3}\left(\tau_{0}^{-}\right)+\Delta \eta_{3}, \quad \eta_{4}\left(\tau_{0}^{+}\right)=\eta_{4}\left(\tau_{0}^{-}\right)+\Delta \eta_{4} \\
& \Delta \eta_{1}=-\alpha_{2}\left\{\eta_{1}\left(\tau_{0}^{-}\right)-\eta_{3}\left(\tau_{0}^{-}\right)\right\} \\
& \Delta \eta_{2}=-\alpha_{2}\left\{y_{1}\left(\tau_{0}^{-}\right)+2 \gamma y_{4}\left(\tau_{0}^{-}\right)-\cos v \tau_{0}^{-}\right\} \\
& \\
& \times\left\{\eta_{1}\left(\tau_{0}^{-}\right)-\eta_{3}\left(\tau_{0}^{-}\right)\right\} / y_{2}\left(\tau_{0}^{-}\right) \\
&-y_{4}\left(\tau_{0}^{-}\right)-\alpha_{2}\left\{\eta_{2}\left(\tau_{0}^{-}\right)-\eta_{4}\left(\tau_{0}^{-}\right)\right\} \\
& \Delta \eta_{3}= \alpha_{3}\left\{\eta_{1}\left(\tau_{0}^{-}\right)-\eta_{3}\left(\tau_{0}^{-}\right)\right\} \\
& \Delta \eta_{4}= \alpha_{3}\left\{y_{1}\left(\tau_{0}^{-}\right)+2 \gamma y_{2}\left(\tau_{0}^{-}\right)-\cos v \tau_{0}^{-}\right\} \\
& \times\left\{\eta_{1}\left(\tau_{0}^{-}\right)-\eta_{3}\left(\tau_{0}^{-}\right)\right\} / y_{2}\left(\tau_{0}^{-}\right) \\
&-y_{4}\left(\tau_{0}^{-}\right)+\alpha_{3}\left\{\eta_{2}\left(\tau_{0}^{-}\right)-\eta_{4}\left(\tau_{0}^{-}\right)\right\}
\end{aligned}
$$

In this paper, damping ratio $\gamma=0.05$, coefficient of restitution $e=0.8$, and mass ratio $\mu=0.1$ are used. Moreover, the relationship between non-dimension clearance $\Delta$ in this paper and the non-dimension clearance $\Delta^{\prime}$ in Masri and Caughey ${ }^{(1)}$ is $\Delta^{\prime}=2 \Delta$.

Results obtained by this method and that of Masri and Caughey ${ }^{(1)}$ are compared as follows: First, the boundaries of stability of the solution with two collisions per period are shown in Table 3. The convergence accuracy of a solution is $\varepsilon=1 \times 10^{-9} . \lambda_{1,2}$ and $\lambda_{3,4}$ in the table show the characteristic multipliers obtained by this method, and the absolute values are shown in Table 3. It turns out from the table that the value of $v$ to which the absolute value of a characteristic multiplier exceeds 1 and the stability of a solution changes is very well in agreement to 10 figures of 
Table 3 Boundary of stability $(\Delta=4.0, \gamma=0.05, e=0.8, \mu=$ $0.1)$

\begin{tabular}{|l|c|c|}
\hline \multicolumn{1}{|c|}{$v$} & $\left|\lambda_{1,2}\right|$ & $\left|\lambda_{3,4}\right|$ \\
\hline 1.0852502 & 0.7595633989 & 0.9999978701 \\
\hline 1.08525018 & 0.7595633939 & 0.9999994107 \\
\hline 1.085250173 & 0.7595633922 & 0.9999999494 \\
\hline 1.085250172 & 0.7595633919 & 1.000000025 \\
\hline 1.08525017 & 0.7595633914 & 1.000000181 \\
\hline 1.0852501 & 0.7595633739 & 1.000005573 \\
\hline 1.085250173 & Stable & $\begin{array}{c}\text { Boundary of } \\
\text { Stability } \\
\text { By Masri and }_{\text {Caughey's }} \\
\text { Method }\end{array}$ \\
\hline 1.085250172 & Unstable \\
\hline
\end{tabular}

Table 4 Boundary of stability by Masri and Caughey's Method

\begin{tabular}{|c|c|c|c|}
\hline \multicolumn{4}{|c|}{$\begin{array}{c}\text { Method of Masri and Caughey } \\
\left(\Delta^{\prime}=8.0, \quad v=1.0852 \sim 2.0\right)\end{array}$} \\
\hline 1.0852 & Unstable & 1.6735 & Unstable \\
\hline 1.0853 & Stable & 1.6736 & Stable \\
\hline$\downarrow$ & & $\downarrow$ & \\
\hline 1.3699 & Stable & 2.0000 & Stable \\
\hline 1.3700 & Unstable & \\
\hline
\end{tabular}

the stable and unstable boundary value of the Masri and Caughey's method ${ }^{(1)}$. This means that processing of variational equation at the time of a collision is also exact in a two-degree-of-freedom impact system.

The result of angular velocity of external force $v=$ $1.0852-2.0$ by the Masri and Caughey's method ${ }^{(1)}$ is shown in Table 4. It is determined also that these boundaries of stability are in agreement with those obtained by our shooting method. On the other hand, Fig. 8 is a figure shown in the paper of Masri and Caughey ${ }^{(1)}$. The hatched stable regions calculated by Masri and Caughey ${ }^{(1)}$ themselves and those calculated by the shooting method which are shown by the arrows are not in agreement at three places in Fig. 8. Popplewell et al. ${ }^{(2)}$ also mentioned that the stability of solutions calculated by Masri and Caughey themselves was wrong. As mentioned above, it is found that although the solution and the method to judge the stability of solutions which Masri and Caughey ${ }^{(1)}$ proposed are correct, but their numerical computation result is wrong.

Calculation times to obtain steady state solutions were also compared in the two-degree-of freedom system. Adopting only the first figure of convergence solution 1/1$\mathrm{O} 2$ at $v=1.1$ and $\Delta=4.0$ in Fig. 8 as the initial value, the solution of $v=1.1$ was calculated, then the periodic solutions of 1/1-O2 were calculated continuously to $v=1.3$ in increasing divisions of 0.01 . The times taken in the calculation are compared in Table 5. Here, the convergence accuracy of a solution was set to $\varepsilon=1 \times 10^{-10}$. RKG-

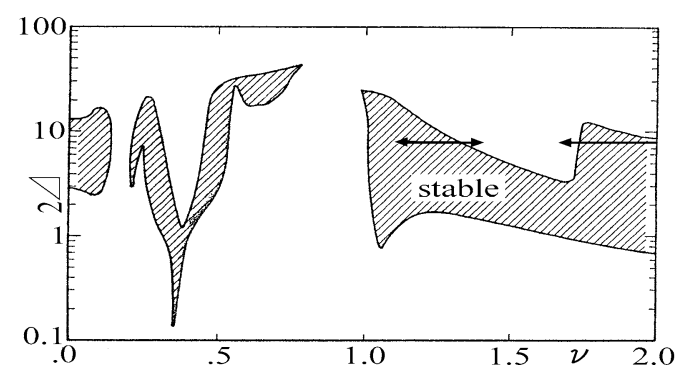

Fig. 8 Stable regions (Uת) calculated by Masri and Caughey ${ }^{(1)}$ and those $(\longleftrightarrow)$ cluculated by the shooting method $(v=0-2.0)$

Table 5 Comparison of calculation time $(\Delta=4.0)$

\begin{tabular}{|c|c|c|c|}
\hline$v$ & Method of calculation & $\begin{array}{c}\text { Initial number } \\
\text { of division }\end{array}$ & $\begin{array}{c}\text { Calculation } \\
\text { time (s) }\end{array}$ \\
\hline \multirow{3}{*}{$\begin{array}{c}1.1 \\
\downarrow\end{array}$} & RKG - Not shooting & \multirow{2}{*}{512} & 4.33 \\
1.3 & RKG+Shooting & & 0.62 \\
\cline { 2 - 2 } & Exact - Not shooting & \multirow{2}{*}{64} & 1.68 \\
\cline { 2 - 2 } & Exact+Shooting & & 0.13 \\
\hline
\end{tabular}

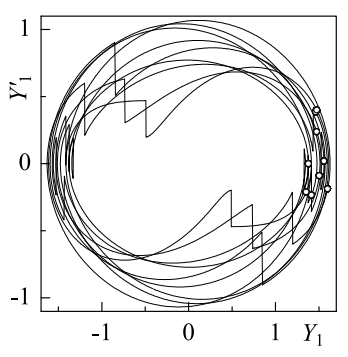

(a) $\quad 1 / 9-\mathrm{E} 18(v=0.5199)$

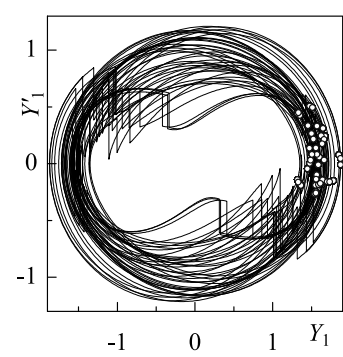

(b) $1 / 43-\mathrm{E} 86(v=0.5464)$
Fig. 9 Phase planes of periodic solutions $(\Delta=4.0)$

Not Shooting method takes the most time. The Exact-Not Shooting method and the RKG+Shooting method follows in that order, and the Exact+Shooting method can calculate solutions in the shortest time. The RKG+Shooting method can calculate in only one-seventh of the time taken in the RKG-Not Shooting method, which does not correct the initial value. The Exact+Shooting method can calculate periodic solutions in a calculation time of one-fifth of that in the RKG+Shooting method. It is verified also in the two-degree-of-freedom system that the Exact+Shooting method is a very high-speed method.

The phase planes of periodic solutions are shown in Fig. 9. Small circles in the figures show the instances when the phase of external force are zero. These complicated periodic solutions are easily obtained using the shooting method.

Moreover, chaos is judged using the Lyapunov exponent. The typical Poincare section of a beat vibration is shown in Fig. 10 (a), and four Lyapunov exponents $\rho$ are written below the figure. Poincare section in the case of chaos is shown in Fig. 10 (b). In the creation of the Poincare section, each solution is calculated only by con- 


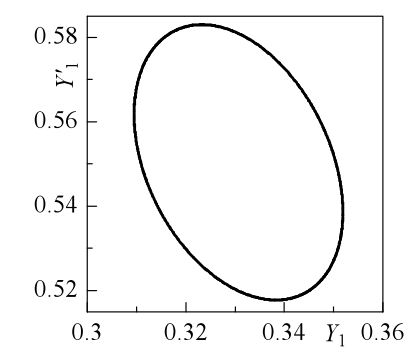

( $\rho=0.00003,0.0003,-0.15,-0.15)$

(a) Beat $(v=1.370)$

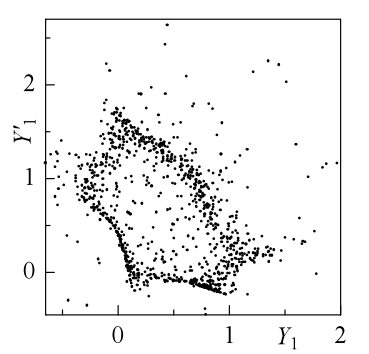

( $\rho=0.03,-0.002,-0.12,-0.21)$

(b) Chaos $(v=1.389)$
Fig. 10 Poincare section and Lyapunov exponents $(\Delta=4.0)$

necting analytical solutions. It is said that one of Lyapunov exponents $\rho$ is zero in the beat and one of those is positive in Chaos. On the other hand, the calculated Lyapunov exponents in Fig. 10 (a) have two extremely small positive values in the beat. However, as the significant figure of Lyapunov exponents are thought to be 2-3 figures below a decimal point ${ }^{(20)}$, this positive value is the numerical calculation error. Therefore, it can be said that the results of the Poincare section and those of Lyapunov exponents correspond well in the figures. It is said that the processing of variational equations in a colliding point is correct, and Lyapunov exponents are also correctly calculated in the two-degree-of-freedom impact system.

\section{Conclusion}

The results of this study are summarized as follows:

(1) A shooting method for impact systems was developed.

(2) By comparing the existing solutions of singleand two-degree-of-freedom systems, it was proved that the periodic solutions and those stabilities obtained by the shooting method were correct.

(3) It was shown that a characteristic multiplier changed discontinuously in a grazing bifurcation produced by generation of a collision, and the grazing bifurcation was explained from a viewpoint of discontinuity in stability of solution.

(4) It was shown that this shooting method is economical in calculation speed and can obtain complicated periodic solutions.

( 5 ) The calculation method of the Lyapunov exponent for the impact systems was shown and numerical results showed that accurate values were acquired.

\section{References}

( 1 ) Masri, S.F. and Caughey, T.K., On the Stability of the Impact Damper, Trans. ASME, J. Appl. Mech., Vol.33 (1966), pp.586-592.

( 2 ) Popplewell, N., Bapat, C.N. and Mclachlan, K., Stable Periodic Vibroimpacts of an Oscillator, J. Sound Vib., Vol.146, No.3 (1983), pp.41-59.

( 3 ) Yoshitake, Y. and Sueoka, A., Quenching of Self-
Excited Vibrations by an Impact Damper, Trans. Jpn. Soc. Mech. Eng., (in Japanese), C, Vol.60, No.569 (1994), pp.50-56.

( 4 ) Moore, J.J., Palazzolo, A.B., Gadangi, G., Nale, T.A., Klusman, S.A., Brown, G.V. and Kascak, A.F., A Forced Response Analysis and Application of Impact Dampers to Rotordynamic Vibration Suppression in a Cryogenic Environment, Trans. ASME, J. Vib. and Acoustics, Vol.117 (1995), pp.300-310.

( 5 ) Collette, F.S., A Combined Tuned Absorber and Pendulum Impact Damper under Random Excitation, J. Sound Vib., Vol.216, No.2 (1998), pp.199-213.

( 6 ) Inoue, J., Araki, Y. and Miyaura, S., On the SelfSynchronization of Mechanical Vibrators: Part 5, Shock Vibrator, Trans. Jpn. Soc. Mech. Eng., (in Japanese), Vol.42, No.353 (1976), pp.103-110.

( 7 ) Han, I. and Lee, Y., Chaotic Dynamics of Repeated Impacts in Vibratory Bowl Feeders, J. Sound Vib., Vol.249, No.3 (2002), pp.529-541.

( 8 ) Meisner, S. and Campbell, B., Development of Gear Rattle Analytical Simulation Methodology, SAE Paper No.951317, (1995), pp.681-690.

( 9 ) Chikatani, Y. and Suehiro, A., Reduction of Idling Rattle Noise in Trucks, SAE Paper No.911044, (1991), pp.49-56.

(10) Azeez, M.F.A. and Vakakis, F.V., Numerical and Experimental Analysis of a Continuous Overhung Rotor Undergoing Vibro-Impacts, Non-Linear Mechanics, Vol.34 (1999), pp.415-435.

(11) Suzuki, K., Aoki, S. and Ohyama, T., Dynamic Response Analysis of the Piping System considering Nonlinear Characteristics of Support (1st Report, Influence of Gap of Support), Trans. Jpn. Soc. Mech. Eng., (in Japanese), C, Vol.53, No.490 (1987), pp.1141-1146.

(12) Horiuchi, T., Impact Vibration Analysis of Group of Hexagonal Bars Immersed in Liquid, Trans. Jpn. Soc. Mech. Eng., (in Japanese), C, Vol.60, No.576 (1994), pp.2691-2698.

(13) Kawai, R. and Kotera, T., Theoretical Research on Vibroimpacts (1st Report, Periodic Impact Motion and Its Stability of Single-Degree-of-Freedom System), Trans. Jpn. Soc. Mech. Eng., (in Japanese), Vol.35, No.274 (1978), pp.1233-1241.

(14) Kobrinskii, A.E., Dynamics of Mechanisms with Elastic Connections and Impact Systems, (1969), p.254, Iliffe Book Ltd., London.

(15) Aprille, T.J,Jr. and Trick, T.N., A Computer Algorithm to Determine the Steady-State Response of Nonlinear Oscillators, IEEE Trans. on Circuit Theory CT19, No.4 (1972), pp.354-360.

(16) Oestreich, M., Hinrichs, N. and Popp, K., Bifurcation and Stability Analysis for a Non-Smooth Friction Oscillator, Archive of Applied Mechanics, Vol.66 (1996), pp.301-314.

(17) Sundararajan, P. and Noah, S.T., Dynamics of Forced Non-Linear Systems Using Shooting/Arclength Continuation Method-Application to Rotor Systems, Trans. ASME, J. Vib. and Acoustics, Vol.119 (1997), pp.9-20.

(18) Yoshitake, Y., Sueoka, A., Tamura, H. and Hai, T., Direct Numerical Integral Method to Determine Peri- 
odic Solutions of Nonlinear Systems (Equations of Motion with Discontinuous Functions), Trans. Jpn. Soc. Mech. Eng., (in Japanese), C, Vol.59, No.561 (1993), pp.1428-1435.

(19) Yoshitake, Y., Sueoka, A., Tamura, S., Kohira, S. and Miyuki, T., Shooting Method Using Analytical Solutions for a Nonlinear System with Piecewise Linear Characteristics, Trans. Jpn. Soc. Mech. Eng., (in Japanese), C, Vol.66, No.641 (2000), pp.23-30.

(20) Shimada, I. and Nagashima, T., A Numerical Approach to Ergodic Problem of Dissipative Dynamical Systems, Prog. Theor, Phys., Vol.61, No.6 (1979), pp.16051616.

(21) Luo, A.C.J. and Han, R.P.S., The Dynamics of a Bouncing Ball with a Sinusoidally Vibrating Table Revisited, Nonlinear Dynamics, Vol.10 (1996), pp.1-18.

(22) Imamura, H. and Suzuki, K., Dynamic Behavior in
Vibro-Impact Mechanical System (Bifurcation Phenomena of $1 / n$ Subharmonic Solutions and Chaotic Behavior), Trans. Jpn. Soc. Mech. Eng., (in Japanese), C, Vol.55, No.510 (1989), pp.267-274.

(23) Foale, S. and Bishop, S.R., Bifurcations in Impact Oscillations, Nonlinear Dynamics, Vol.6 (1994), pp.285299.

(24) Yoshitake, Y. and Sueoka, A., Forced Self-Excited Vibration with Dry Friction, Applied Nonlinear Dynamics and Chaos of Mechanical Systems with Discontinuities, Chap.10, edited by Wiercigroch, M. and Kraker, B., (2000), pp.237-259, World Scientific.

(25) Yoshitake, Y., Sueoka, A., Tamura, H. and Hai, T., Vibrations of Nonlinear Systems with Discontinuities (The Case of a Preloaded Compliance System), JSME Int. J., Ser. C, Vol.41, No.4 (1997), pp.710-717. 